\title{
The GPD program at COMPASS
}

\author{
Paweł Sznajder* (on behalf of the COMPASS Collaboration) \\ National Centre for Nuclear Research, Warsaw \\ E-mail: pawel.sznajder@fuw.edu.pl
}

In this paper we summarize recent measurements of exclusive reactions at COMPASS, which are a part of the GPD program. New results on the transverse target spin asymmetries for exclusive $\rho^{0}$ production are presented. Some of these asymmetries are sensitive to the GPDs $E$, which are related to the orbital angular momentum of quarks. Other asymmetries are sensitive to the chiral-odd, transverse GPDs $H_{T}$. Planned measurements of Deeply Virtual Compton Scattering, which are a part of the approved COMPASS-II proposal, are also discussed. The main aim of these measurements is access to the GPDs $H$, which are related to the 3-dimensional nucleon tomography.

Photon 2013,

20-24 May 2013

Paris, France

* Speaker. 


\section{Introduction}

Hard exclusive electro- and muoproduction of photons or mesons on nucleons has played an important role in studies of the hadron structure and recently gained renewed interest as it allows access to generalised parton distributions (GPDs). GPDs provide a novel and comprehensive description of the partonic structure of the nucleon and contain a wealth of new information. For instance GPDs give a description of the nucleon as an extended object, referred to as 3-dimensional nucleon tomography, and give access to the orbital angular momentum of quarks. Deeply Virtual Compton Scattering (DVCS) is the most straightforward process to access quarks GPDs, while mesons, due to their different quark contents and quantum numbers, can be used to access GPDs for specific quark flavours and gluons.

At leading twist, the chiral-even GPDs $H$ and $E$ are sufficient to describe exclusive production of photons or mesons on a spin $1 / 2$ target. These GPDs are of special interest as they are related to the total angular momentum carried by partons in the nucleon [1]. GPDs $H$ are well constrained in accessible $x_{B j}$ range by JLAB, HERA and HERMES. Constraints on GPDs $E$ are weak and come mainly from measurements of nucleon Pauli form factors.

There exist also chiral-odd, "transverse" GPDs, in particular $H_{T}$ and $\bar{E}_{T}$. It was shown [2] that they are required to describe exclusive $\pi^{+}$production on transversely polarized protons.

In this paper we summarize recent measurement of the transverse target spin asymmetries for exclusive $\rho^{0}$ production on protons. The asymmetries are sensitive both to chiral-even and chiralodd GPDs. The interpretation of results is done in the framework of the GPD model proposed by Goloskokov and Kroll [3]. Also, we give projections for measurements of DVCS process on unpolarized protons in COMPASS-II. The main aim of these measurements is access to the GPDs $H$, which are related to the 3-dimensional nucleon tomography.

\section{Transverse target spin asymmetries for exclusive $\rho^{0}$ production}

\subsection{Formalism}

For exclusive meson production off a transversely polarized target five single (UT) and three double (LT) spin asymmetries can be defined. These are

$$
\begin{array}{lll}
A_{\mathrm{UT}}^{\sin \left(\phi-\phi_{s}\right)}=-\frac{\operatorname{Im}\left(\sigma_{++}^{+-}+\epsilon \sigma_{00}^{+-}\right)}{\sigma_{0}}, & A_{\mathrm{LT}}^{\cos \left(\phi-\phi_{s}\right)}=\frac{\operatorname{Re} \sigma_{++}^{+-}}{\sigma_{0}}, & A_{\mathrm{UT}}^{\sin \left(\phi+\phi_{s}\right)}=-\frac{\operatorname{Im} \sigma_{+-}^{+-}}{\sigma_{0}}, \\
A_{\mathrm{UT}}^{\sin \left(2 \phi-\phi_{s}\right)}=-\frac{\operatorname{Im} \sigma_{+0}^{-+}}{\sigma_{0}}, & A_{\mathrm{LT}}^{\cos \left(2 \phi-\phi_{s}\right)}=-\frac{\operatorname{Re} \sigma_{+0}^{++}}{\sigma_{0}}, & A_{\mathrm{UT}}^{\sin \left(3 \phi-\phi_{s}\right)}=-\frac{\operatorname{Im} \sigma_{+-}^{-+}}{\sigma_{0}} \\
A_{\mathrm{UT}}^{\sin \phi_{s}}=-\frac{\operatorname{Im} \sigma_{+0}^{+-}}{\sigma_{0}}, & A_{\mathrm{LT}}^{\cos \phi_{s}} & =-\frac{\operatorname{Re} \sigma_{+0}^{+-}}{\sigma_{0}} .
\end{array}
$$

The photoabsorption cross sections or the interference terms $\sigma_{m n}^{i j}$ are proportional to the bilinear combinations of the helicity amplitudes $\mathcal{M}$ for the photoproduction subprocess $\gamma^{*} p \rightarrow \rho^{0} p$,

$$
\sigma_{m n}^{i j} \propto \sum \mathcal{M}_{m^{\prime} i^{\prime}, m i}^{*} \mathcal{M}_{m^{\prime} i^{\prime}, n j},
$$

where the helicity of the virtual photon is denoted by $m, n=-1,0,+1$ and the helicity of the initialstate proton is given by $i, j=-1 / 2,+1 / 2$. The sum runs over all spin combinations for the final state, 
given by the spin of the meson $m^{\prime}=-1,0,+1$ and the spin of the final-state proton $i^{\prime}=-1 / 2,+1 / 2$. For brevity a dependence on the kinematic variables is omitted here.

The total unpolarized cross section, $\sigma_{0}$, is given by the sum of cross sections for longitudinally, $\sigma_{L}$, and transversely, $\sigma_{T}$, polarized virtual photons,

$$
\sigma_{0}=\frac{1}{2}\left(\sigma_{++}^{++}+\sigma_{++}^{--}\right)+\epsilon \sigma_{00}^{++}=\sigma_{L}+\epsilon \sigma_{T},
$$

and the virtual photon polarization parameter can be approximated by $\epsilon \simeq(1-y) /\left(1-y+y^{2} / 2\right)$.

Each asymmetry is related with specific modulation of the cross section in $\phi$ and $\phi_{s}$ angles indicated by the superscript. The angle $\phi$ is the azimuthal angle between the lepton plane, given by the momenta of the incoming and the scattered leptons, and the hadron plane, given by the momenta of the virtual photon and the meson. The angle $\phi_{s}$ is the azimuthal angle between the lepton plane and the spin direction of the target nucleon. Full formula for the cross section can be found in Ref. [4].

The asymmetries are extracted from data selected as described in the following.

\subsection{Event selection}

To determine the transverse target spin asymmetries for exclusive production of $\rho^{0}$ meson the data taken in 2007 and 2010 with polarized protons were analysed. Each selected event contains a primary vertex with only one incoming and one outgoing muon track and with only two outgoing hadron tracks of opposite charges. It is assumed, that the outgoing hadrons are pions. The $\rho^{0}$ resonance is selected by the cut on the reconstructed invariant mass $0.5 \mathrm{GeV} / c^{2}<M_{\pi \pi}<1.1 \mathrm{GeV} / c^{2}$. Because recoiled target particle in undetected, the exclusivity is checked by the missing energy, $E_{\text {miss }}=\left((p+q-v)^{2}-p^{2}\right) / 2 M_{p}$, where $M_{p}$ is the mass of the proton and $p, q$ and $v$ are the fourmomenta of proton, photon and meson, respectively. For exclusive events the reconstructed values of $E_{\text {miss }}$ are close to zero. To select these events the cut $-2.5 \mathrm{GeV}<E_{\text {miss }}<2.5 \mathrm{GeV}$ is used. The cut $0.05(\mathrm{GeV} / c)^{2}<p_{T}^{2}<0.5(\mathrm{GeV} / c)^{2}$ is also applied, where $p_{T}^{2}$ is the squared transverse momentum of $\rho^{0}$ with respect to the virtual photon direction. The lower cut on $p_{T}^{2}$ suppresses a contribution from the coherent production on the target nuclei, while the upper cut provides a further reduction of non-exclusive background.

The kinematic region is defined by the following cuts: $1(\mathrm{GeV} / c)^{2}<Q^{2}<10(\mathrm{GeV} / c)^{2}, 0.1<$ $y<0.9,0.003<x_{B j}<0.35, W>5 \mathrm{GeV} / c^{2}$ (invariant mass of the virtual photon - nucleon system) and $p_{T}^{2}$ cuts as indicated above. The asymmetries were extracted using the $2 \mathrm{D}$ binned likelihood method after subtraction of remaining semi-inclusive background. Details of the analysis can be found in Ref. [5].

\subsection{Results and discussion}

The mean values of measured asymmetries are shown in Fig. 1. They are given for the mean values of kinematic variables, $\left\langle Q^{2}\right\rangle=2.2(\mathrm{GeV} / c)^{2},\left\langle x_{B j}\right\rangle=0.039,\left\langle p_{T}^{2}\right\rangle=0.2(\mathrm{GeV} / c)^{2}$, $\langle W\rangle=8.1 \mathrm{GeV} / c^{2}$ and $\langle y\rangle=0.24$, of the selected data set. The asymmetry $A_{U T}^{\sin \phi_{s}}$ was found to be $-0.019 \pm 0.008$ (stat) \pm 0.003 (sys). All other asymmetries were found to be small, consistent with zero within experimental uncertainty. The asymmetries measured as a function of $Q^{2}, x_{B j}$ or $p_{T}^{2}$ 


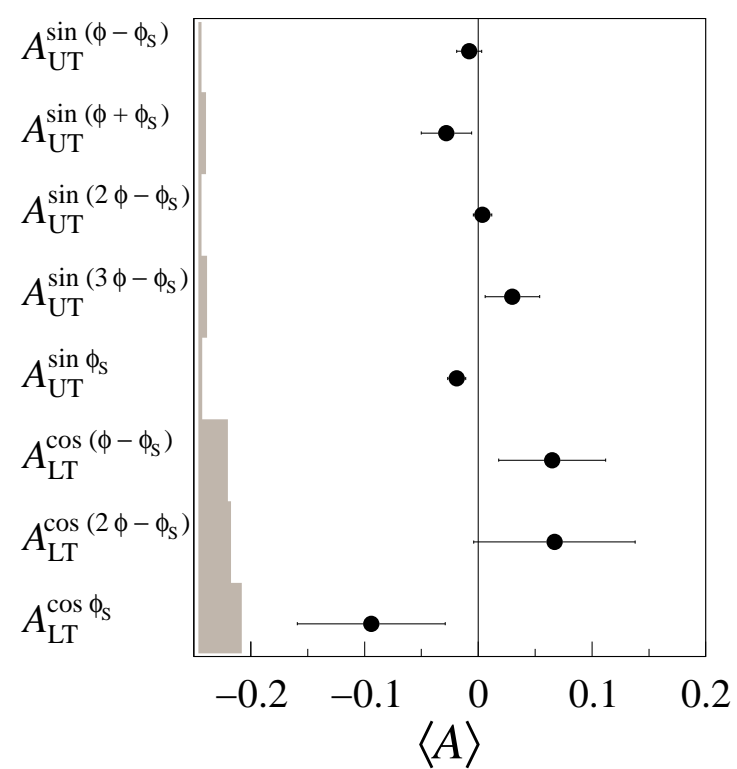

Figure 1: Mean values of azimuthal asymmetries for a transversely polarized proton target. The error bars (left bands) represent the statistical (systematic) uncertainties. Mean values of kinematic variables are indicated in the text.

can be found in Ref. [5], together with a comparison with predictions of the GPD model proposed by Goloskokov and Kroll [4]. The model agrees well with our data.

For an interpretation of results in the framework of the model of particular interest are the following asymmetries, for which the dependence on the helicity amplitudes reads

$$
\begin{aligned}
\sigma_{0} A_{U T}^{\sin \left(\phi-\phi_{s}\right)} & =-2 \operatorname{Im}\left[\epsilon \mathcal{M}_{0-, 0+}^{*} \mathcal{M}_{0+, 0+}+\mathcal{M}_{+-,++}^{*} \mathcal{M}_{++,++}+\frac{1}{2} \mathcal{M}_{0-,++}^{*} \mathcal{M}_{0+,++}\right], \\
\sigma_{0} A_{U T}^{\sin \left(2 \phi-\phi_{s}\right)} & =-\operatorname{Im}\left[\mathcal{M}_{0+,++}^{*} \mathcal{M}_{0-, 0+}\right], \\
\sigma_{0} A_{U T}^{\sin \phi_{s}} & =-\operatorname{Im}\left[\mathcal{M}_{0-,++}^{*} \mathcal{M}_{0+, 0+}-\mathcal{M}_{0+,++}^{*} \mathcal{M}_{0-, 0+}\right] .
\end{aligned}
$$

The dominant contribution from the $\gamma_{L}^{*} \rightarrow \rho_{L}^{0}$ transition is given by $\mathcal{M}_{0+, 0+}$ and $\mathcal{M}_{0-, 0+}$ helicity amplitudes, which are related to chiral-even GPDs $H$ and $E$, respectively. The suppressed contribution from the $\gamma_{T}^{*} \rightarrow \rho_{T}^{0}$ transition is given by $\mathcal{M}_{++,++}$and $\mathcal{M}_{+-,++}$helicity amplitudes, which are also related to chiral-even GPDs. Description of the $\gamma_{T}^{*} \rightarrow \rho_{L}^{0}$ transition is possible by inclusion of chiral-odd GPDs $H_{T}$ and $\bar{E}_{T}$, which are related to $\mathcal{M}_{0-,++}$ and $\mathcal{M}_{0+,++}$ helicity amplitudes, respectively. The $\gamma_{L}^{*} \rightarrow \rho_{T}^{0}$ and $\gamma_{T}^{*} \rightarrow \rho_{-T}^{0}$ transitions are known to be suppressed and are neglected in this formalism.

The vanishing $A_{U T}^{\sin \left(\phi-\phi_{s}\right)}$ asymmetry is interpreted as a cancellation of GPDs $E^{u}$ and $E^{d}$ due to their different sign but similar magnitude. A contribution of chiral-odd GPDs is negligible here, as one can see from comparison of calculations of Refs. [3] and [6]. The $A_{U T}^{\sin \phi_{s}}$ asymmetry represents an imaginary part of two bilinear products of helicity amplitudes. The first product is related with GPDs $H$ and $H_{T}$, while the second one is related with GPDs $E$ and $\bar{E}_{T}$. The latter product appears also in the $A_{U T}^{\sin \left(2 \phi-\phi_{s}\right)}$ asymmetry. The $A_{U T}^{\sin \phi_{s}}$ asymmetry is found to be different from zero, while the $A_{U T}^{\sin \left(2 \phi-\phi_{s}\right)}$ asymmetry vanishes. It implies non-negligible contribution of GPDs $H_{T}$. It is the first 
experimental evidence from hard exclusive $\rho^{0}$ production for the observation of these chiral-odd GPDs.

In preparation is a measurement of azimuthal asymmetries for exclusive $\omega$ production. The comparison between $\rho^{0}$ and $\omega$ is of special interest, since they probe different combinations of GPDs for $u$ and $d$ quarks. In particular, for $\omega$ the $A_{U T}^{\sin \left(\phi-\phi_{s}\right)}$ asymmetry is expected to be $\approx-0.1$ [6].

\section{Deeply Virtual Compton Scattering at COMPASS-II}

Measurement of Deeply Virtual Compton Scattering (DVCS) on unpolarized proton target is a part of COMPASS-II proposal [7], which has been approved by CERN Research Bord on December 2010. The proposal includes two years of data taking for the GPD program (planned for 2016-2017), preceded by a short pilot program, which was successfully taken at the end of 2012. Projections for measurements included in the proposal are based on assumptions confirmed by a short data taking in 2009, where the first DVCS events were observed. The measurements with the transversely polarized $\mathrm{NH}_{3}$ target are considered for the future. They will be proposed as an addendum to the proposal.

For the GPD program at COMPASS-II the apparatus has been optimized for measurements of exclusive reactions. In addition to the two electromagnetic calorimeters (ECAL1 and ECAL2), a new large angle electromagnetic calorimeter ECAL0 is being constructed to cover high $x_{B j}$ region for the DVCS measurement, with $1 / 3$ ready in 2012. Also, a $2.5 \mathrm{~m}$ long liquid hydrogen target surrounded by a $4 \mathrm{~m}$ long recoil proton detector (CAMERA) has been build.

The COMPASS spectrometer is located at the unique CERN SPS M2 beam line, which can deliver high energy muon beams $(50 \mathrm{GeV}-280 \mathrm{GeV})$ of both charges and polarizations. It allows to probe region of intermediate $x_{B j}$, where both cross section of DVCS and its interference with competitive Bethe-Heitler process can be measured. This region is of special interest for the nucleon tomography, since both gluons/sea-quarks and valence quarks can be probed. COMPASS is the only experiment which can explore this kinematic region in the near future, before availability of new colliders.

The cross section for single photon production, which includes contributions from both BetheHeitler and DVCS processes as well as the interference terms, reads

$$
d \sigma=d \sigma^{B H}+d \sigma_{\text {unpol }}^{D V C S}+P_{\mu} d \sigma_{\text {pol }}^{D V C S}+e_{\mu} a^{B H} R e T^{D V C S}+e_{\mu} P_{\mu} a^{B H} I m T^{D V C S},
$$

where $P_{\mu}$ and $e_{\mu}$ are the polarization and the charge (in units of the elementary charge) of the polarized muon beam. The interference between Bethe-Heitler and DVCS arises on the level of their amplitudes, $a^{B H}$ and $T^{D V C S}$, respectively. For brevity a dependence on the kinematic variables and the $\phi$ angle is omitted here. The $\phi$ angle is the azimuthal angle between the lepton plane and the hadron plane, similarly as in the case of $\rho^{0}$ analysis.

Combining two measurements of the cross section performed with muon beams of opposite charges and polarizations, $d \sigma_{\leftarrow}^{+}$and $\sigma_{\rightarrow}^{-}$, one can get the cross section sum and difference

$$
\begin{aligned}
& S_{C S, U}=d \sigma_{\leftarrow}^{+}+\sigma_{\rightarrow}^{-}=2\left(d \sigma^{B H}+d \sigma_{\text {unpol }}^{D V C S}+e_{\mu} P_{\mu} a^{B H} \operatorname{Im} T^{D V C S}\right), \\
& D_{C S, U}=d \sigma_{\leftarrow}^{+}-\sigma_{\rightarrow}^{-}=2\left(P_{\mu} d \sigma_{\text {pol }}^{D V C S}+e_{\mu} a^{B H} \operatorname{ReT}^{D V C S}\right),
\end{aligned}
$$




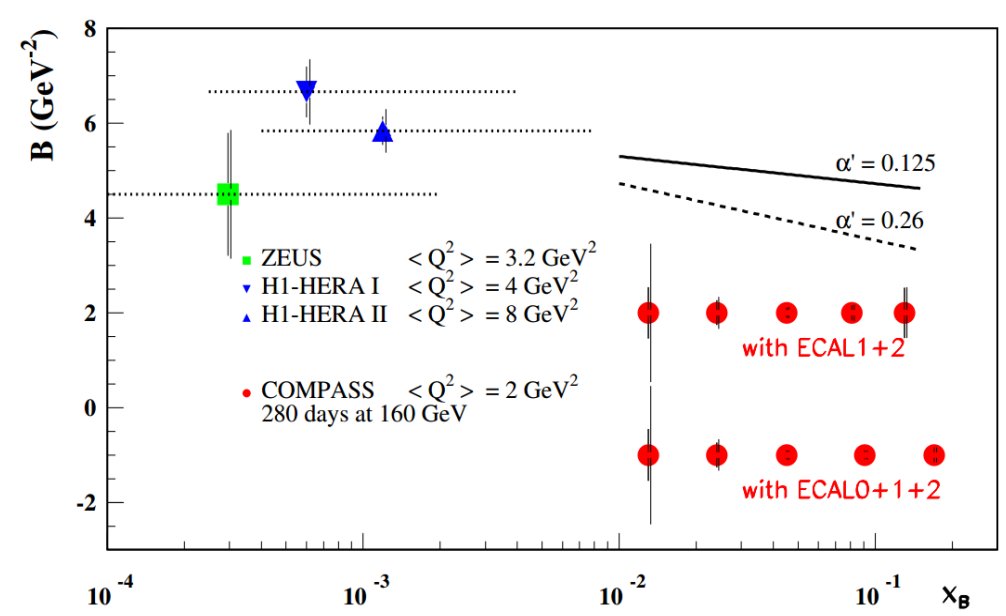

Figure 2: The projection of measurement of slope $b$ for DVCS process for 2016-2017 data using only ECAL1 and ECAL2 (first row) and also ECAL0 (second row). Existing data points from ZEUS in a similar $Q^{2}$ range as well as various assumed $x_{B j}$ dependences of the slope [7] are also shown.

where $C S$ indicates that both lepton charge and lepton spin are reversed simultaneously when changing from $\mu^{+}$to $\mu^{-}$, while $U$ denotes the unpolarized target.

The dependence on $\phi$ angle for terms related with DVCS process reads

$$
\begin{aligned}
d \sigma_{\text {unpol }}^{D V C S S} & \propto \mathbf{c}_{\mathbf{0}}^{\mathbf{D V C S}}+c_{1}^{D V C S} \cos \phi+c_{2}^{D V C S} \cos 2 \phi, \\
d \sigma_{\text {pol }}^{D V C S} & \propto s_{1}^{D V C S} \sin \phi, \\
I m T^{D V C S} & \propto \mathbf{s}_{\mathbf{1}}^{\text {int }} \sin \phi+s_{2}^{\text {int }} \sin 2 \phi, \\
R e T^{D V C S} & \propto \mathbf{c}_{\mathbf{0}}^{\text {int }}+\mathbf{c}_{\mathbf{1}}^{\text {int }} \cos \phi+c_{2}^{\text {int }} \cos 2 \phi+c_{3}^{i n t} \cos 3 \phi,
\end{aligned}
$$

where $s$ and $c$ coefficients are related to certain combinations of Compton Form Factors (CFF). Bold-faced are the leading twist elements.

After integration of $S_{C S, U}$ over $\phi$ angle and subtraction of the BH contribution it can be used to access DVCS unpolarized cross section. The slope of $t$ distribution of this cross section is of special interest, as it is related to the transverse size of nucleon and thus important for the nucleon tomography. The projection for measurement of $t$ slope for DVCS process in different bins of $x_{B j}$ at COMPASS-II is shown in Fig. 2.

The $S_{C S, U}$ and $D_{C S, U}$, non-integrated over $\phi$ angle, can be used to access CFF $\mathcal{H}$ from $s_{1}^{\text {int }} \propto \operatorname{Im}\left(F_{1} \mathcal{H}\right)$ and $c_{1}^{\text {int }} \propto \operatorname{Re}\left(F_{1} \mathcal{H}\right)$, respectively. Here $F_{1}$ is the Dirac form factor of the proton. The projection for a measurement of $D_{C S, U}$ cross section difference as a function of $\phi$ angle at COMPASS-II is shown in Fig. 3.

\section{Summary}

The five single-spin azimuthal asymmetries for unpolarized beam and three double-spin azimuthal asymmetries for longitudinally polarized beam were extracted for exclusive $\rho^{0}$ muoproduction on transversely polarized protons. The asymmetry $A_{U T}^{\sin \phi_{s}}$ was found to be $-0.019 \pm 0.008($ stat $) \pm$ 0.003 (sys). This result indicates observation of non-vanishing "transverse" GPDs $H_{T}$. All other 


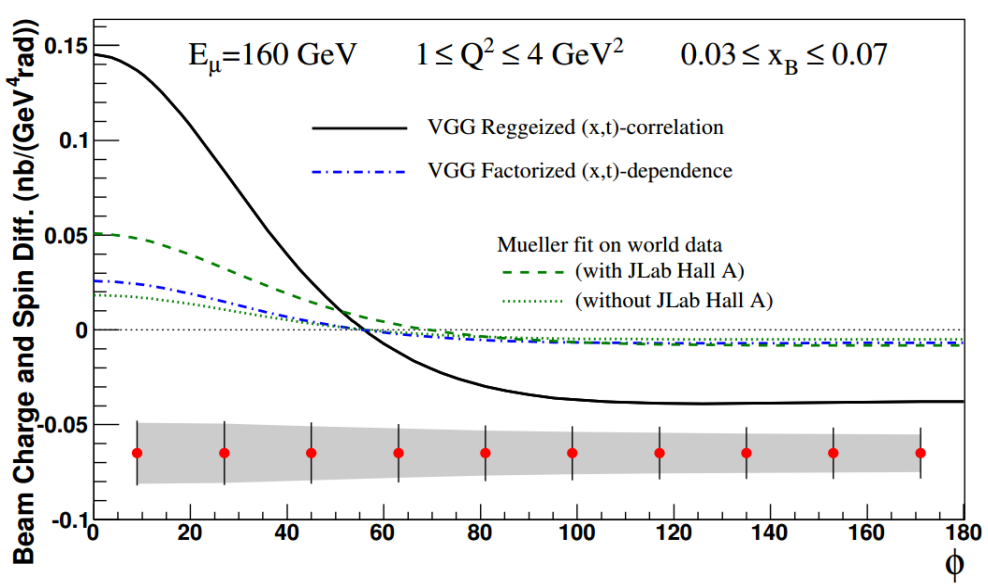

Figure 3: The projection of measurement of $D_{C S, U}$ cross section difference as a function of $\phi$ angle for 2016-2017 data together with predictions of various models for DVCS [7].

asymmetries were found to be small, consistent with zero within experimental uncertainty. The GPD model proposed by Goloskokov and Kroll [3] describes well our results.

A new proposal for the COMPASS-II experiment [7] has been approved. Future GPD studies are a substantial part of this proposal. The use of a new detector, a large Recoil Proton Detector, will allow a clean selection of the sample of exclusive events for the studies of the DVCS and DVMP processes. The measurements with the unpolarized liquid hydrogen target are foreseen first, while the measurements with the transversely polarized $\mathrm{NH}_{3}$ target are considered for the future.

\section{References}

[1] X. Ji, Phys. Rev. Lett. 78 (1997) 610.

[2] S.V. Goloskokov and P. Kroll, Eur. Phys. J. C65 (2010) 137.

[3] S.V. Goloskokov and P. Kroll, submitted to Eur. Phys. J. C [arXiv:1310.1472].

[4] M. Diehl and S. Sapeta, Eur. Phys. J. C41 (2005) 515.

[5] The COMPASS Collaboration, submitted to Phys. Lett. B [arXiv:1310.1454].

[6] S.V. Goloskokov and P. Kroll, Eur. Phys. J. C59 (2009) 809.

[7] The COMPASS Collaboration, CERN-SPSC-2010-014. 